\title{
A NOTE ON THE ALGEBRA OF MEASURABLE OPERATORS OF AN $A W^{*}$-ALGEBRA
}

\author{
S. K. BERBERIAN
}

(Received Jan. 22, 1970)

1. Introduction. This note is concerned with the relation between an $A W^{*}$. algebra $A$ and the algebra $M$ of 'measurable operators' affiliated with $A$ [4], the general idea being that properties of $A$ are reflected in analogous properties of the larger algebra $M$. We consider here two specific properties: (1) the property of being a Baer*-ring, and (2) the monotonicity of the square root operation on positive elements.

More precisely, to say that $A$ is an $A W^{*}$-algebra means that $A$ is both a $C^{*}$-algebra and a Baer*-ring. K. Saito has shown that $M$ is also a Baer ${ }^{*}$-ring ([4], Theorem 6.4); we offer here a simpler proof, of a nominally more general result. The algebra $A$ has the property that $0 \leqq a \leqq b$ implies $a^{1 / 2} \leqq b^{1 / 2}$ (this is true in any $C^{*}$-algebra); assuming the set of self-adjoint elements of $A$ has a certain 'monotone convergence property' (automatically verified when $A$ is a von Neumann algebra), we show that $M$ has the property that $0 \leqq x \leqq y$ implies $x^{1 / 2} \leqq y^{1 / 2}$. The latter result plays a role in D. Topping's theory of vector lattices of self-adjoint operators ([5], see p. 27, Proposition 10).

2. The Baer*-ring property. Let $A$ be an $A W^{*}$-algebra and let $M$ be the algebra of measurable operators affiliated with $A$ [4]. We extract as axioms the properties of $A$ and $M$ that are relevant for the discussion in this section: (1) $M$ is a *-algebra with unity element 1 , (2) $A$ is a *-subalgebra of $M$, (3) if $x, y \in M$ and $x^{*} x+y^{*} y=1$, then $x, y \in A$ ([4], Lemma 5.2), (4) $A$ is a Baer ${ }^{*}$-ring in the sense of I. Kaplansky [3], and (5) for any $x \in M, 1+x^{*} x$ is invertible and $\left(1+x^{*} x\right)^{-1} \in A([4]$, Lemmas $4.1,5.2)$.

Taking (1)-(5) as axioms, we show in this section that $M$ is also a Baer*-ring. We write

$$
a_{x}=\left(1+x^{*} x\right)^{-1} \quad(x \in M) .
$$

Note that $x^{*} x=0$ implies $x=0$. \{Proof : $1=0+1=x^{*} x+1^{*} 1$, therefore $x \in A$ by (3), hence $x=0$ by a property of Baer ${ }^{*}$-rings ([3], p. 31, Theorem 21). $\}$ Also, $M$ has no new partial isometries (i. e., none not already in $A$ ), hence no new projections. 
\{Proof : If $w^{*} w=e, e$ a projection, then $1=e+(1-e)=w^{*} w+(1-e)^{*}(1-e)$, therefore $w \in A$ by (3).\} It follows that the set of projections of $M$ (i. e., of $A$ ) is a complete lattice ([3], p.29, Theorem 19). Every element of $A$ has a right projection ([3], p. 28); so does every element of $M$ :

LEMMA 2.1. If $x \in M$ and $e=R P\left(1-a_{x}\right)$ (the right projection of $1-a_{x}$, calculated in $A)$, then $x e=x$ and $e$ is the smallest such projection.

PROOF. If $g$ is a projection, the following conditions imply one another: $x g=0, x^{*} x g=0,\left(1+x^{*} x\right) g=g, g=\left(1+x^{*} x\right)^{-1} g=a_{x} g,\left(1-a_{x}\right) g=0, e g=0$. The largest such projection is $g=1-e$, thus $1-e$ is the largest projection such that $x(1-e)=0$; that is, $e$ is the smallest projection such that $x e=x$.

With notation as in Lemma 2.1, we write $e=R P(x)$; that is, $R P(x)$ is defined to be $R P\left(1-a_{x}\right)$. We also define $L P(x)=R P\left(x^{*}\right)$; thus, $L P(x)$ is the smallest projection $f$ such that $f x=x$. By definition,

$$
L P(x)=R P\left(x^{*}\right)=R P\left(1-a_{x^{*}}\right) .
$$

LEMMA 2.2. If $x, y \in M, e=R P(x)$ and $g=L P(y)$, then $x y=0$ iff $e g=0$.

ProOF. If $e g=0$ then $x y=(x e)(g y)=0$. Conversely, if $x y=0$ then $x^{*} x y y^{*}=0$, $\left(1+x^{*} x\right) y y^{*}=y y^{*}, y y^{*}=\left(1+x^{*} x\right)^{-1} y y^{*}=a_{x} y y^{*},\left(1-a_{x}\right) y y^{*}=0,\left(1-a_{x}\right)\left(1+y y^{*}\right)$ $=1-a_{x}, 1-a_{x}=\left(1-a_{x}\right)\left(1+y y^{*}\right)^{-1}=\left(1-a_{x}\right) a_{y^{*}}, \quad\left(1-a_{x}\right)\left(1-a_{y^{*}}\right)=0$; since $A$ is a Baer*-ring this implies $\left[R P\left(1-a_{x}\right)\right]\left[R P\left(1-a_{y^{*}}\right)\right]=0$ (for a self-adjoint element, we need not distinguish between $L P$ and $R P$ ), that is, $R P(x) L P(y)=0$.

Generalizing Saitô's theorem for the case that $A$ is an $A W^{*}$-algebra ([4], Theorem 6.4), we have :

THEOREM 2.3. If $A$ and $M$ satisfy axioms (1)-(5), then $M$ is a Baer*-ring.

Proof. Let $S$ be any subset of $M$ and write $R(S)=\{y \in M: S y=0\}$ for the right-annihilator of $S$; the problem is to show that the right ideal $R(S)$ is generated by a projection. Define $e=\sup \{R P(x): x \in S\}$. In view of Lemma 2.2, the following conditions imply one another: $y \in R(S), x y=0$ for all $x \in S$, $R P(x) L P(y)=0$ for all $x \in S, e L P(y)=0, e y=0,(1-e) y=y, y \in(1-e) M$. Thus $R(S)=(1-e) M$.

3. Monotonicity of square roots. Let $A$ be an $A W^{*}$-algebra, $M$ the 
algebra of measurable operators of $A$. In the application [5], $A$ is assumed to be of finite class; we do not make this restriction here, but, for the reader who is interested only in the finite case, we cite alternative references to [1].

LEMMA 3.1. If $x \in M, x \geqq 0$ and $x$ is invertible in $M$, then $x^{-1} \geqq 0$.

PROOF. Write $x=z^{2}$, with $z$ self-adjoint ([4], Corollary 5.2; [1], Corollary 6.2). Then $z$ is invertible and $x^{-1}=\left(z^{-1}\right)^{2}=\left(z^{-1}\right)^{*}\left(z^{-1}\right) \geqq 0$.

LEMMA 3.2. If $a \in A, 0 \leqq a \leqq 1$, and a has an inverse in $M$, then $a^{-1} \geqq 1$.

PROOF. Same as ([2], p. 179, Lemma 2).

LEMMA 3. 3. If $x$ and $y$ are invertible elements of $M$ such that $0 \leqq x \leqq y$, then $x^{-1} \geqq y^{-1} \geqq 0$.

Proof. By Lemma 3.1, $y^{-1} \geqq 0$. Write $x=s^{2}, y=t^{2}$, with $s$ and $t$ selfadjoint, and set $w=s t^{-1}$. Then $w^{*} w=t^{-1} s^{2} t^{-1}=\left(t^{-1}\right)^{*} x t^{-1} \leqq\left(t^{-1}\right)^{*} y t^{-1}=t^{-1} y t^{-1}=1$, thus $w \in A,\|w\| \leqq 1$ ([4], Lemma $5.2 ;[1]$, Lemma 5.1). Since $w$ is invertible in $M$, so is $w^{*} w$; citing Lemma 3.2 we have $1 \leqq\left(w^{*} w\right)^{-1}=w^{-1}\left(w^{-1}\right)^{*}=t s^{-1} s^{-1} t$ $=t x^{-1} t$, therefore $y^{-1}=t^{-1} 1 t^{-1} \leqq t^{-1}\left(t x^{-1} t\right) t^{-1}=x^{-1}$.

LEMMA 3. 4. If $x, y \in M, 0 \leqq x \leqq y, x y=y x$, and $y \geqq \varepsilon 1$ for some real number $\varepsilon>0$, then $x^{1 / 2} \leqq y^{1 / 2}$.

Proof. We can suppose $\varepsilon=1$. Then $y=1+\left[(y-1)^{1 / 2}\right]^{2}$ shows that $y$ is invertible ([4], Lemma 4.1; [1], Corollary 3.1), and $y^{-1} \leqq 1$ by Lemma 3. 3. Write $x=s^{2}, y=t^{2}$, with $s \geqq 0$ and $t \geqq 0, s \in\{x\}^{\prime \prime}, t \in\{y\}^{\prime}$ ([4], Corollary 5.2; [1], Corollary 6.2); since $x y=y x$ it follows that $s t=t s$, therefore

$$
0 \leqq y-x=t^{2}-s^{2}=(t+s)(t-s) .
$$

Since $\left(t^{-1}\right)^{*} t^{-1}=\left(t^{2}\right)^{-1}=y^{-1} \leqq 1$, we have $t^{-1} \in A$ and $0 \leqq t^{-1} \leqq 1$, therefore $t \geqq 1$ by Lemma 3.2 ; then $t+s \geqq t \geqq 1$ shows that $t+s$ is invertible. Let $z=(t+s)^{-1}$; by Lemma $3.1, z \geqq 0$. Clearly $z$ commutes with $t$ and $s$, and therefore with $t^{2}-s^{2}$ $=y-x \geqq 0$; since the product of commuting positives is positive $\{\mathrm{e}$.g., in the above notation, $\left.x y=s^{2} t^{2}=t s^{2} t=(s t)^{*}(s t) \geqq 0\right\}$ it follows that $z(y-x) \geqq 0$, i.e., in view of $(*), 0 \leqq z(t+s)(t-s)=t-s$.

LEMMA 3.5. If $x, y \in M$ and $0 \leqq x \leqq y$, then $x^{1 / 2} \leqq(y+\varepsilon 1)^{1 / 2}$ for every real number $\varepsilon>0$. 
PROOF. Since $x \leqq x+\varepsilon 1$, where $x$ and $x+\varepsilon 1$ commute and $x+\varepsilon 1 \geqq \varepsilon 1$, Lemma 3.4 yields

$$
x^{1 / 2} \leqq(x+\varepsilon 1)^{1 / 2}
$$

On the other hand, $\varepsilon 1 \leqq x+\varepsilon 1 \leqq y+\varepsilon 1$, where $x+\varepsilon 1$ and $y+\varepsilon 1$ are invertible (see the proof of Lemma 3. 4), therefore $0 \leqq(y+\varepsilon 1)^{-1} \leqq(x+\varepsilon 1)^{-1} \leqq \varepsilon^{-1} 1$ by Lemma 3. 3 ; moreover, $(y+\varepsilon 1)^{-1},(x+\varepsilon 1)^{-1} \in A$, therefore $(y+\varepsilon 1)^{-1 / 2} \leqq(x+\varepsilon 1)^{-1 / 2}$ by the monotonicity of square roots in a $C^{*}$-algebra, and Lemma 3.3 then yields

$$
(x+\varepsilon 1)^{1 / 2} \leqq(y+\varepsilon 1)^{1 / 2} .
$$

Combining ( i), (ii), we obtain the desired inequality.

The $A W^{*}$-algebra $A$ is said to have the monotone convergence property if it satisfies the following condition: if $a_{n}$ is a sequence of self-adjoint elements in $A$ such that $a_{1} \leqq a_{2} \leqq a_{3} \leqq \cdots$ and $a_{n} \leqq b(n=1,2,3, \cdots)$ for some self-adjoint element $b$ of $A$, then sup $a_{n}$ exists (with respect to the usual ordering of selfadjoints). The notation $a_{n} \uparrow a$ means that $a_{1} \leqq a_{2} \leqq a_{3} \leqq \cdots, \sup a_{n}$ exists, and $a=\sup a_{n}$. We remark that $a$ also serves as a supremum for the $a_{n}$ in $M$. \{The point is that if $x$ is a self-adjoint element of $M$ such that $a_{n} \leqq x$ for all $n$, then $a \leqq x$. To prove this, it suffices to consider the case that $a_{1} \geqq 0$. Then $1 \leqq 1+a_{n} \uparrow 1+a$ implies that $1 \geqq\left(1+a_{n}\right)^{-1} \downarrow(1+a)^{-1}$. Also, $1 \leqq 1+a_{n} \leqq 1+x$ implies, by Lemma 3. 3, that $1 \geqq\left(1+a_{n}\right)^{-1} \geqq(1+x)^{-1}$. Writing $b=(1+a)^{-1}$, we have $(1+x)^{-1} \leqq \inf \left(1+a_{n}\right)^{-1}$ $=b$, therefore $1+x \geqq b^{-1}, x \geqq b^{-1}-1=a$. $\}$

The foregoing definition makes sense also for $M$ : we say that $M$ has the monotone convergence property iff sup $x_{n}$ exists for any increasing sequence of self-adjoint elements of $M$ such that $x_{n} \leqq y(n=1,2,3, \cdots)$ for some self-adjoint $y$ in $M$.

LEMMA 3.6. If $A$ has the monotone convergence property, then so does $M$.

PROOF. Suppose $x_{n}, y \in M$ are self-adjoint, $x_{1} \leqq x_{2} \leqq x_{3} \leqq \cdots$, and $x_{n} \leqq y$ for all $n$. Subtracting $x_{1}$ throughout, we can suppose $x_{1} \geqq 0$. Then

$$
1 \leqq 1+x_{1} \leqq 1+x_{2} \leqq \cdots \leqq 1+y,
$$

therefore by Lemma 3. 3,

$$
1 \geqq\left(1+x_{1}\right)^{-1} \geqq\left(1+x_{2}\right)^{\text {ㄴ }} \geqq \cdots \geqq(1+y)^{-1} \geqq 0 .
$$


Since $\left(1+x_{n}\right)^{-1} \in A$, we may set $z=\inf \left(1+x_{n}\right)^{-1}$, and it follows readily from Lemma 3. 3 that $x_{n} \uparrow z^{-1}-1$. A An essential point is that $z$ is invertible. Indeed, $z \geqq(1+y)^{-1} \geqq 0$. Setting $t=(1+y)^{1 / 2}$, we have $t^{2}=1+y$ invertible, hence $t$ is invertible, and $t^{*} z t \geqq t^{*}(1+y)^{-1} t=t\left(t^{2}\right)^{-1} t=1$; thus $t z t=t^{*} z t$ is invertible, hence so is $\left.t^{-1}(t z t) t^{-1}=z\right\}$.

We remark that the foregoing considerations can be cast more generally in terms of directed families (of any cardinality); the directed family formulation of Lemma 3. 6 generalizes an earlier result for algebras of finite class ([2], p. 179, Corollary).

LEMMA 3.7. If $A$ has the monotone convergence property, and $y$ is any positive element of $M$, then

$$
y^{1 / 2}=\inf _{\mathbf{c}>0}(y+\varepsilon 1)^{1 / 2} .
$$

PROOF. By Lemma 3.5 we have $y^{1 / 2} \leqq(y+\varepsilon 1)^{1 / 2}$ for all $\varepsilon>0$.

Consider the sequence $y_{n}=y+(1 / n) 1 \quad(n=1,2,3, \cdots)$; thus $y_{1} \geqq y_{2} \geqq y_{3} \geqq \cdots$ $\geqq y \geqq 0$. By Lemma 3. 4,

$$
\left(y_{1}\right)^{1 / 2} \geqq\left(y_{2}\right)^{1 / 2} \geqq \cdots \geqq y^{1 / 2} ;
$$

in view of Lemma 3.6, we may set $z=\inf \left(y_{n}\right)^{1 / 2}$, and it will clearly suffice to show that $z=y^{1 / 2}$.

We note first that $z \in\{y\}^{\prime \prime}$. \{Proof : Assuming $s \in\{y\}^{\prime}$ we are to show that $z s=s z$. Since $\{y\}$ is a ${ }^{*}$-subalgebra of $M$, we can suppose $s$ is self-adjoint. If $u$ is the Cayley transform of $s$ ([4], Theorem 5.1; [1], Theorem 4.1), one has $\{s\}^{\prime}=\{u\}^{\prime}$, thus it will suffice to show that $z u=u z$. Since $\{u\}^{\prime \prime}=\{s\}^{\prime \prime} \subset\{y\}^{\prime \prime \prime}=\{y\}^{\prime}=\left\{y_{n}\right\}$, we have $\left(y_{n}\right)^{1 / 2} \in\left\{y_{n}\right\}^{\prime \prime} \subset\{u\}^{\prime \prime \prime}=\{u\}^{\prime}$, that is, $u$ commutes with $\left(y_{n}\right)^{1 / 2}$; then

$$
u z u^{*}=u\left[\inf \left(y_{n}\right)^{1 / 2}\right] u^{*}=\inf \left[u\left(y_{n}\right)^{1 / 2} u^{*}\right]=\inf \left(y_{n}\right)^{1 / 2}=z,
$$

as was to be shown.\}

Since $\left(y_{n}\right)^{1 / 2} \geqq y^{1 / 2}$ for all $n$, we have $z \geqq y^{1 / 2}$; since, moreover, $z$ commutes with $y^{1 / 2}$ (by the preceding paragraph) it follows that $z^{2} \geqq y$. \{Indeed, $z^{2}-y$ $=\left(z+y^{1 / 2}\right)\left(z-y^{1 / 2}\right)$ is the product of commuting positives. $\}$ On the other hand, $z \leqq\left(y_{n}\right)^{1 / 2}$ for all $n$, and since $z$ commutes with $\left(y_{n}\right)^{1 / 2}$ it follows that $z^{2} \leqq y_{n}$; thus

$$
0 \leqq z^{2}-y \leqq y_{n}-y=(1 / n) 1
$$

therefore $z^{2}-y \in A$ and $\left\|z^{2}-y\right\| \leqq 1 / n$ for all $n$, i. e., $z^{2}-y=0, z=y^{1 / 2}$. 
THEOREM 3.8. If $A$ has the monotone convergence property, and if $x, y \in M$ satisfy $0 \leqq x \leqq y$, then $x^{1 / 2} \leqq y^{1 / 2}$.

PROOF. For any $\varepsilon>0$, Lemma 3.5 yields $x^{1 / 2} \leqq(y+\varepsilon 1)^{1 / 2}$; in view of Lemma 3.7, this implies $x^{1 / 2} \leqq y^{1 / 2}$.

For the case that $A$ is of finite class, Theorem 3.8 evolved in correspondence (1963) with Professor Topping, to whom I am indebted for several of the key ideas.

REMARK. In Lemma 3.3, the assumption that $y$ is invertible is redundant; that is, if $y \geqq x \geqq 0$ and $x$ is invertible, then $y$ is also invertible. \{Proof: By Lemma 3.1 we may write $x^{-1}=t^{2}$ with $t^{*}=t$; then $t y t \geqq t x t=1$, therefore $t y t$ is invertible, hence so is $t^{-1}(t y t) t^{-1}=y$.

\section{REFERENCES}

[1] S. K. Berberian, The regular ring of a finite $A W^{*}$-algebra, Ann. of Math., (2) 65(1957), 224-240.

[2] S. K. Berberian, Note on a theorem of Fuglede and Putnam, Proc. Amer. Math. Soc., 10(1959), 175-182.

[ 3 ] I. KAPLANSKY, Rings of operators, W. A. Benjamin, Inc., New York, 1968.

[4] K. SAITô, On the algebra of measurable operators for a general $A W^{*}$-algebra, Tohoku Math. J., 21(1969), 249-270.

[5] D. M. TOPPING, Vector lattices of self-adjoint operators, Trans. Amer. Math. Soc., 115(1965), 14-30.

DEPARTMENT OF MATHEMATICS

THE UNIVERSITY OF TEXAS

Austin, Texas, U.S. A. 